\title{
Aneurysmal Coronary Artery Fistulae: How Big is Big Enough?
}

\author{
Abhishek Jaiswal, MBBS ${ }^{1}$ Chong H. Park, MD ${ }^{1}$ \\ 1 Division of Cardiology, Department of Internal Medicine, \\ Cardiovascular Center, New York Hospital Queens/Weill Medical \\ College of Cornell University, New York, New York
}

\begin{abstract}
Address for correspondence Abhishek Jaiswal, MBBS, Cardiovascular Center, WA 200, 5645 Main Street, Flushing, New York, NY 11365 (e-mail: jais.abhishek@gmail.com).
\end{abstract}

Int J Angiol 2014;23:139-142.

\begin{abstract}
Keywords

- coronary artery fistula

- coronary angiography

- coronary aneurysm

- transcatheter interventions

- coils

Coronary artery fistula (CAF) is the most common congenital anomaly of coronary arteries. Management strategies for CAF causing symptoms and large shunting are well described. With growing use of noninvasive imaging modalities, the diagnosis of asymptomatic CAF has increased. This poses challenge on the management of CAF with high-risk features as therapeutic strategy of incidentally found CAF remains open to debate. The actual risk of complications in such cases is mere speculations based on small studies and need to be revisited. We describe the case of an asymptomatic 78year-old woman with a large saccular coronary aneurysm associated with coronary artery to pulmonary artery fistula that was treated successfully using coils. We discuss the updated literature on the management of CAF as well.
\end{abstract}

Coronary artery fistula (CAF) is a rare anomaly through which coronary blood flow is usually shunted into either a cardiac chamber, great vessel, or other structures, bypassing the myocardial capillary network. Usually, it is detected as an incident finding during coronary angiography, other noninvasive thoracic imaging or occasionally, incidental finding of a continuous murmur, characteristically heard over the left sternal border and at the apex might be present. The detection has recently increased because of the widespread use of diagnostic modalities including coronary angiography, multidetector computed tomography, and magnetic resonance imaging for other diseases. ${ }^{1}$

It is reported to be present in approximately $0.2 \%$ of routine coronary angiograms. ${ }^{2}$ However, symptomatic cases with complications such as high-output heart failure, pulmonary hypertension, myocardial ischemia, and infective endocarditis have been reported. Treating physicians are usually on crossroads in cases with asymptomatic detection because of lack of consensus on the management strategy. Data on presumed low sudden death rate in asymptomatic patients are limited because of lack of large studies with long followups. With increasing detection of asymptomatic cases, it is prudent to revise our traditional approach. In this article, we cross-examine the conventional approach with different views and suggest an alternative strategy by eliciting our case.

\section{Case Report}

A 78-year-old hypertensive woman with episodic chest pressure was referred to our cardiac catheterization laboratory for angiographic evaluation. Patient recently immigrated to United States in her usual health status 3 months ago. She started developing present symptoms 2 months before her clinic visit and initially attributed these symptoms to her underlying chronic acid reflux. Progression of symptoms infrequency and severity led to follow-up visit and was referred to emergency room for further evaluation. Patient had a prior coronary angiographic evaluation for similar symptoms 5 years ago in her native country, and she was told to have normal findings. However, results were unobtainable.

Physical examination revealed a blood pressure of 158/ $78 \mathrm{~mm} \mathrm{Hg}$, and a regular pulse of 86 beats per minute. Electrocardiography showed nonspecific ST-T wave changes. The cardiologist decided to proceed with coronary angiography that revealed mild luminal irregularities and preserved left ventricular systolic function. In addition, angiography also showed a left anterior descending coronary artery to main pulmonary trunk fistula with an in-between saccular aneurysm. The aneurysm was $2.0 \times 2.5 \mathrm{~cm}$ in maximum dimension (-Fig. 1). The CAF feeding this aneurysm was less than $2 \mathrm{~mm}$ in diameter. A right heart catheterization was 


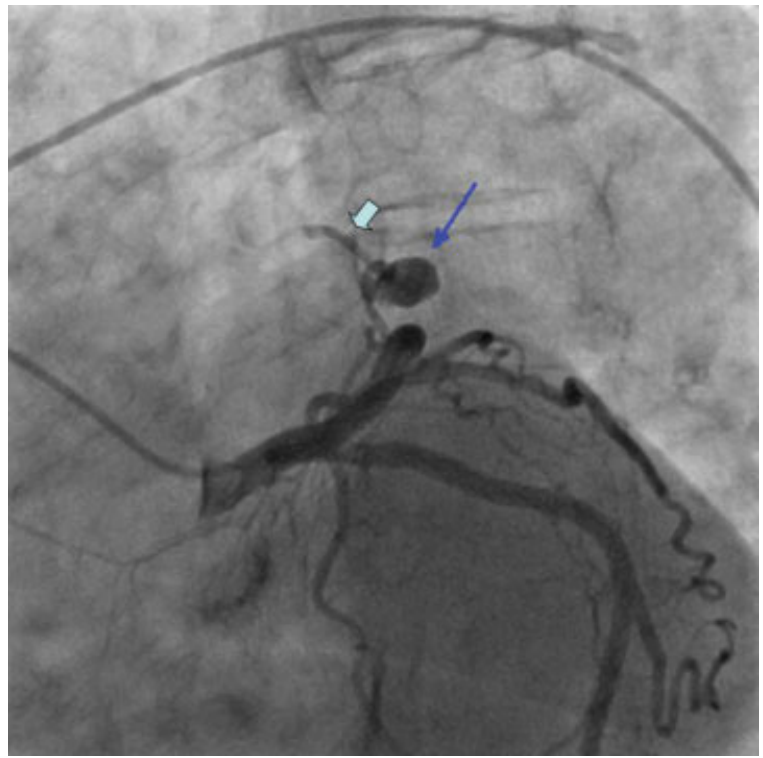

Fig. 1 Coronary angiogram showing the aneurysmal sac at the end of coronary injection (arrow). Arrowhead showing the termination of fistula connection near the pulmonary artery.

performed to further evaluate any systemic-to-pulmonary shunt. There was no significant left-to-right heart shunt. Right heart hemodynamic study revealed normal pulmonary pressures with pulmonary artery pressure of $36 / 19 \mathrm{~mm} \mathrm{Hg}$ and pulmonary capillary wedge pressure of $12 \mathrm{~mm} \mathrm{Hg}$.

Patient refused any further work-up with velocity encoded cine magnetic resonance imaging, which was recommended for better quantification of the shunt size as proximal location of CAF in the main pulmonary trunk might have influenced angiographic shunt calculation due to streaming of oxygenated blood. Patient was deemed to be at high risk for future complications secondary-to-spontaneous aneurismal rupture on the basis of the large aneurysm diameter to feeder artery diameter ratio and a decision was made for percutaneous intervention. Two Cook coils (Cook Medical Inc., Bloomington, IN) were deployed. Subsequent angiographic images showed little residual blood flow through the aneurysm with a small clot formation and undetectable coronary artery to pulmonary artery blood flow (-Fig. 2). Patient remained asymptomatic after 3 months of follow-up. Her symptom of episodic chest burning was cured by oral proton-pump inhibitor therapy.

\section{Discussion}

Most CAFs are congenital but rarely could these be acquired. ${ }^{3,4}$ Although most remain asymptomatic during childhood and adolescence, many develop symptoms because of complications in adulthood. Aneurysm formation has been reported in around one in five cases. ${ }^{5}$ Rupture of these aneurysms is rare and usually occurs in large ones. Ruptured aneurysm may clinically be presented as chest pain, pericardial effusion, cardiac tamponade, or even sudden cardiac death and could mimic the presentation of acute aortic dissection. ${ }^{6}$

Available treatment options for CAF include surgical ligation either with or without coronary artery bypass surgery,

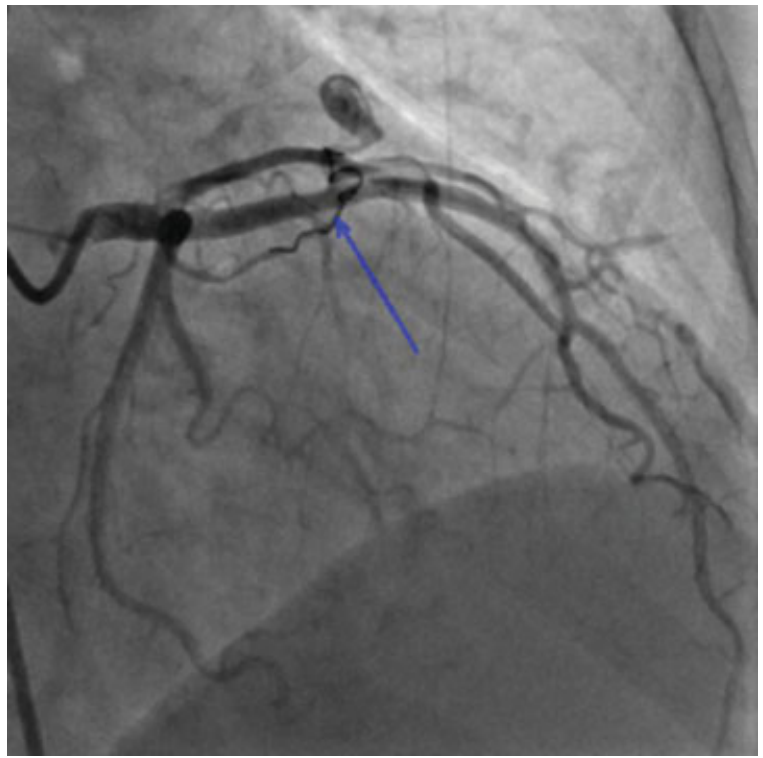

Fig. 2 Arrow showing the coil applied proximal to the aneurysmal sac. There is less blood in the aneurysm (less dense dye) and a possible thrombus inside the sac. Also, there is no blood flow after the aneurysm to the pulmonary artery.

and percutaneous transcatheter closure. It is widely accepted that fistula closure should be considered in symptomatic patients with heart failure, myocardial ischemia, or high shunt ratios to prevent further complications. ${ }^{7}$ However, there is no consensus on the management of asymptomatic aneurysms. ${ }^{8}$ The treatment of large asymptomatic CAF remains controversial and the recommendations are based solely on anecdotal cases, individual experiences, or small retrospective series. ${ }^{9}$ Many believe that giant aneurysms larger than 5-cm warrant intervention. ${ }^{10,11}$

We derive, indirectly, from the data accumulated from studies done on renal artery aneurysm that aneurysm diameter greater than $2 \mathrm{~cm}$ may be associated with complications. $^{12}$ However, because there is no national or international registry of patients with CAF, it would be hard to know the real complication rate. Asymptomatic CAF with small aneurysm should be closely observed and early intervention in those with progressive dilatation during follow-up might be considered. When managed conservatively, prophylactic precautions of subacute bacterial endocarditis are recommended, as bacterial endocarditis is a recognized complication in CAF patients with aneurysm. ${ }^{13}$ Antiplatelet therapy is recommended, especially in patients with distal CAFs and dilated coronary arteries.

We suggest that while discussing management options with asymptomatic patients with large aneurysm, percutaneous transcatheter intervention should be brought up in the discussion because the risk of sudden death in these patients is largely unknown. However, this statement should not be deduced as a justification for an invasive procedure. There is a need to design a national/international registry for further insights into optimal management of CAF especially asymptomatic ones. 


\section{Author Contributions}

Both A.J. and C.H.P. participated in the management of this patient. A.J. wrote the first draft of this manuscript. C.H.P. helped in editing the manuscript.

\section{Conflict of Interest}

None.

\section{Disclosure}

The authors report no financial relationships or conflicts of interest regarding the content herein.

\section{References}

1 Hara H, Moroi M, Araki T, et al. Coronary artery fistula with an associated aneurysm detected by 16-slice multidetector row computed tomographic angiography. Heart Vessels 2005;20(4):184-185

2 Katoh T, Zempo N, Minami Y, et al. Coronary arteriovenous fistulas with giant aneurysm: two case reports. Cardiovasc Surg 1999; 7(4):470-472

3 Sandhu JS, Uretsky BF, Zerbe TR, et al. Coronary artery fistula in the heart transplant patient. A potential complication of endomyocardial biopsy. Circulation 1989;79(2):350-356

4 Patterson MS, Vaina S, Serruys PW. Percutaneous treatment of an iatrogenic cardiac fistula. Catheter Cardiovasc Interv 2008; 72(2):259-262
5 Ghaffari S, Akbarzadeh F, Pourafkari L. Aneurysmal coronary arteriovenous fistula closing with covered stent deployment: a case report and review of literature. Cardiol J 2011;18(5):556-559

6 Misumi T, Nishikawa K, Yasudo M, Suzuki T, Kumamaru H. Rupture of an aneurysm of a coronary arteriovenous fistula. Ann Thorac Surg 2001;71(6):2026-2027

7 Tirilomis T, Aleksic I, Busch T, Zenker D, Ruschewski W, Dalichau H. Congenital coronary artery fistulas in adults: surgical treatment and outcome. Int J Cardiol 2005;98(1):57-59

8 Armsby LR, Keane JF, Sherwood MC, Forbess JM, Perry SB, Lock JE. Management of coronary artery fistulae. Patient selection and results of transcatheter closure. J Am Coll Cardiol 2002;39(6): 1026-1032

9 Testuz A, Roffi M, Bonvini RF. Coronary to pulmonary artery fistulas: an incidental finding with challenging therapeutic options. J Invasive Cardiol 2011;23(7):E177-E180

10 Mawatari T, Koshino T, Morishita K, Komatsu K, Abe T. Successful surgical treatment of giant coronary artery aneurysm with fistula. Ann Thorac Surg 2000;70(4):1394-1397

11 Nakamura K, Shiratori K, Hashimoto K. Giant saccular aneurysm of coronary arteriovenous fistula to the main pulmonary artery: intraoperative assessment by using fluorescent imaging. Ann Thorac Cardiovasc Surg 2010;16(5):354-357

12 Henke PK, Cardneau JD, Welling TH III, et al. Renal artery aneurysms: a 35-year clinical experience with 252 aneurysms in 168 patients. Ann Surg 2001;234(4):454-462, discussion 462-463

13 Said SA. Current characteristics of congenital coronary artery fistulas in adults: A decade of global experience. World J Cardiol 2011;3(8):267-277 